\title{
ESTUDIO DE LA SALUD MENTAL EN ESTUDIANTES UNIVERSITARIOS DE LA RAMA SOCIOSANITARIA
}

\author{
J. Inmaculada Sánchez Casado \\ Universidad de Extremadura \\ iscasado@unex.es \\ Elena I. Benítez Sánchez \\ Universidad de Extremadura \\ ebenitezx@alumnos.unex.es
}

Recepción Artículo: 16 mayo 2021 Admisión Evaluación: 16 mayo 2021 Informe Evaluador 1: 12 mayo 2021 Informe Evaluador 2: 23 mayo 2021 Aprobación Publicación: 01 junio 2021

\section{RESUMEN}

El estigma y los recurrentes prejuicios en torno a la salud mental continúan coexistiendo en la sociedad globalizada del conocimiento y de la comunicación en pleno S.XXI. No olvidemos que la población general suele ser "opaca" ante cualquier problema psíquico y/o psicológico. Se acepta, en el contexto tecnológico actual y más en esta etapa pandémica, todo lo referente a los problemas físicos pero no tanto aquellos referentes a salud mental. De ahí, que la idea-fuerza del presente trabajo se centra en analizar el posible impacto de una salud mental positiva en el grupo de estudiantes universitarios de la rama sociosanitaria. Entendemos que la capacitación y/o formación de estos futuros profesionales ha fomentado un mayor estudio "per se" en cuanto a la necesidad de identificar rasgos vocacionales o de motivación intrínseca para el desempeño profesional, y por ello se ha comprobado en la revisión bibliográfica realizada que experimentan una prevalencia mayor de enfermedades mentales que otros estudiantes universitarios o que la población general.

Además también se ha valorado que los estudiantes de Grado que quieren cursar estas carreras, están más predispuestos a padecer depresión, ansiedad o problemas con el alcohol, y que estas circunstancias tienden a empeorar conforme avanzan en su formación de acuerdo a los 50 artículos analizados. Una cuestión de evolución nada desdeñable por las especiales exigencias que implica tales Grados en la historia de vida de cada estudiante. Por ello, tal situación generalizada de excesiva responsabilidad asociada a su competencia profesionalizadora de toma de decisiones, genera una serie de demandas y necesidades específicas para las instituciones de Educación Superior, que no siempre son adecuadas en su respuesta universitaria. De manera que el conocimiento de dicho fenómeno es vital y no solo porque afecta al posible rendimiento académico del estudiante universitario de ciencias de la salud, sino porque también coarta la producción de conocimiento y contamina su futuro socioprofesional en los términos que plantea la OMS como calidad de vida total.

Palabras clave: salud mental; estudiantes universitarios de ciencias de la salud; revisión bibliográfica 


\section{ABSTRACT}

Study of mental health in university students of the social health Branch. The stigma and the recurrent prejudies around the mental health continues existing in the globalised and well informed society of the XXI century. We cannot forget the opacity presented by the population in the face of any kind of mental health problem. It is well accepted in our tecnological enviroment and mainly in this pandemic stage all the phisical ilnesses, on the contrary mental health is not recognised as an disease by many people in our society. Because of all that, the main end point of this investigation is to analize the possible impact of mental health and a possitive aproach of itself in a students' group of health occupations. It is well known that the capacity and formation of these future professionals has encouraged the research in relation to the necessity of identify vocational roles and intrinsic motivation for the professional performance; all that has been proved in this narrative review, where the students of health occupations show a higher prevalence of mental diseases than other students.

Besides, it has been analize that the students of this areas of knowledge are more inclined to develop depression, anxiety or alcoholic addiction; this situation tends to get worse, as we have seen in more than 50 articles, over the passing time. The scenario is very relevant to consider, mainly because of the high exigencies of this degrees and the lack of valuable response offered by the institutions of Higher Education. As we can see, the knowledge of these cirncunstances is vital, and not only because it affects the student's productivity, it also darkens their professional future in the W.H.O. terms of total "life's quality".

Keywords: mental health; students; health occupations; narrative review

\section{INTRODUCCIÓN}

La salud mental entendida en términos generales como un estado de equilibrio entre una persona y su entorno social \& cultural, que incluye su bienestar emocional psicológico y social, según la OMS, es un tema ampliamente estudiado en la población general. El bienestar mental se ha desarrollado con los años como un componente importante de la salud integral, de la llamada calidad de vida total del sujeto. Sin embargo, todavía se mantienen intactas cuestiones de gran relevancia como: ¿es posible identificar la adecuada salud menta??, o bien, ¿la salud mental se puede mantener a lo largo de toda la vida de la misma manera? y otra de las más difíciles, ¿cómo diferenciar la normalidad de la anormalidad en el mundo actua?? (Valencia Collazos, 2007)

Con las diversas investigaciones realizadas parece cada vez más que la vida de las personas tiene que ver con las ocasiones en que existen momentos de salud mental y otras situaciones en las que debido al estrés emocional o psicosocial, la salud mental se ve afectada; este cambio de paradigma se ve reflejado en la entrevista realizada por el doctor Ahmed Okasha, Presidente de la Asociación Mundial de Psiquiatría, quien consideraba a la salud mental como "un estado de bienestar en el cual los individuos reconocen sus habilidades, son capaces de enfrentarse con los estresantes normales de la vida, pueden trabajar de una manera productiva y fructífera y hacer contribuciones en sus comunidades". (Okasha, 2005) Por todo lo anterior se puede concluir que según estos modelos transaccionales, todos en algún momento podemos estar predispuestos a tener problemas con nuestra salud mental. Todos podemos enfrentarnos a un estado que sobrepase ese equilibrio y precisemos de algún tipo de acompañamiento psicoterapéutico para superarlo.

El problema de su estudio es que hay ciertos grupos de poblaciones "diana" poco representados en dichas investigaciones, quizá por una cuestión social o de perspectiva sociodemográfica, y éstos son, como no podía ser de otra manera, de los jóvenes universitarios. Un colectivo muy especial, filtrado académicamente hablando, llamado a ocupar puestos de responsabilidad, con una función social imprescindible en las sociedades de primer mundo. En esta etapa de la vida, es donde según el saber popular se adquieren experiencias y vivencias que determinarán el futuro relato vital, y por ello existe una específica vulnerabilidad ante las enfermedades mentales, que puede ser desencadenadas por ciertos "triggers" como las exigencias académicas, la separación del ambiente familiar y la vida independiente. (Perales et al., 2019) Si a esto se le añade que un porcentaje nada desdeñable de las enfermedades mentales graves pueden debutar en la adultez temprana, podemos hablar sin dudar de un colectivo de riesgo ad hoc en la sociedad de la 4⿳亠丷冖 revolución tecnológica. 
La salud mental ha empezado a ser analizada desde hace relativamente poco tiempo, quizá la preocupación social por este tipo de situaciones a nivel universitario ha ido aumentando progresivamente; así en los servicios de salud mental en Reino Unido o USA se está reportando un incremento exponencial en el número de estudiantes que presentan problemas que se categorizan como severos. (Brown, 2018) Y ciertamente se puede responsabilizar de este deterioro de la salud mental en los estudiantes, a las exigencias académicas, a las dificultades del desarrollo del aprendizaje o a la adquisición de destrezas que generan situaciones de éxito o de fracaso muy marcadas, y que pueden afectar de lleno a la psique de los universitarios. Vélez, L., \& Gutiérrez, J. (2010)

Aunque está claro que no siempre es sencillo conseguir que los alumnos hablen de sus posibles enfermedades mentales, aquí sigue jugando un papel importante el "estigma social" que puede representar, y las dificultades que puede encontrar el alumnado al revelar sus problemas psicológicos al personal de la institución universitaria. Esta situación revela la especial importancia que pueden tener los mecanismos de ayuda que implanten las distintas universidades, como los programas tutoriales o los servicios de orientación y/o atención psicológica existentes en cada Facultad. (Martin, 2010)

Sin embargo dentro de este colectivo de estudiantes universitarios hay determinados subgrupos más predispuestos que otros a padecer estas enfermedades como se puede apreciar en diversas publicaciones (Cova Solar et al., 2007; Antúnez Sanhueza \& Vinet, 2013) En esa dirección podemos observar una tendencia destacada en los estudiantes que cursan carreras relacionadas con el ámbito de Ciencias de la Salud, entre las que se incluyen Medicina, Psicología, Odontología o Enfermería; destaca especialmente esta primera debido no solo a la amplia duración (ya que cuando se termina la residencia con la especialización ésta sobrepasa la década de duración) sino a causa de las exigencias propias de la carrera, resaltando como problemas principales la ansiedad y la depresión, cuyos valores son superiores a los encontrados en la población general. (Idrugo Jave \& Sanchez Cabrejos, 2020)

La prevalencia de los trastornos mentales varía según la titulación estudiada, y aunque en Grados como el de Medicina se encuentre en primer lugar, seguido de la depresión, (Idrugo Jave \& Sanchez Cabrejos, 2020) en otros tan relacionados como el Grado de Enfermería, la depresión se encuentra en primer lugar seguido de la ansiedad y del estrés. (Rodelo Valle \& Garay Núñez, 2020)

Gráfica 1 Funcionamiento psicológico medio predicho por la cantidad de créditos universitarios y el valor de la red familiar. Fuente: (Walsemann et al., 2015)

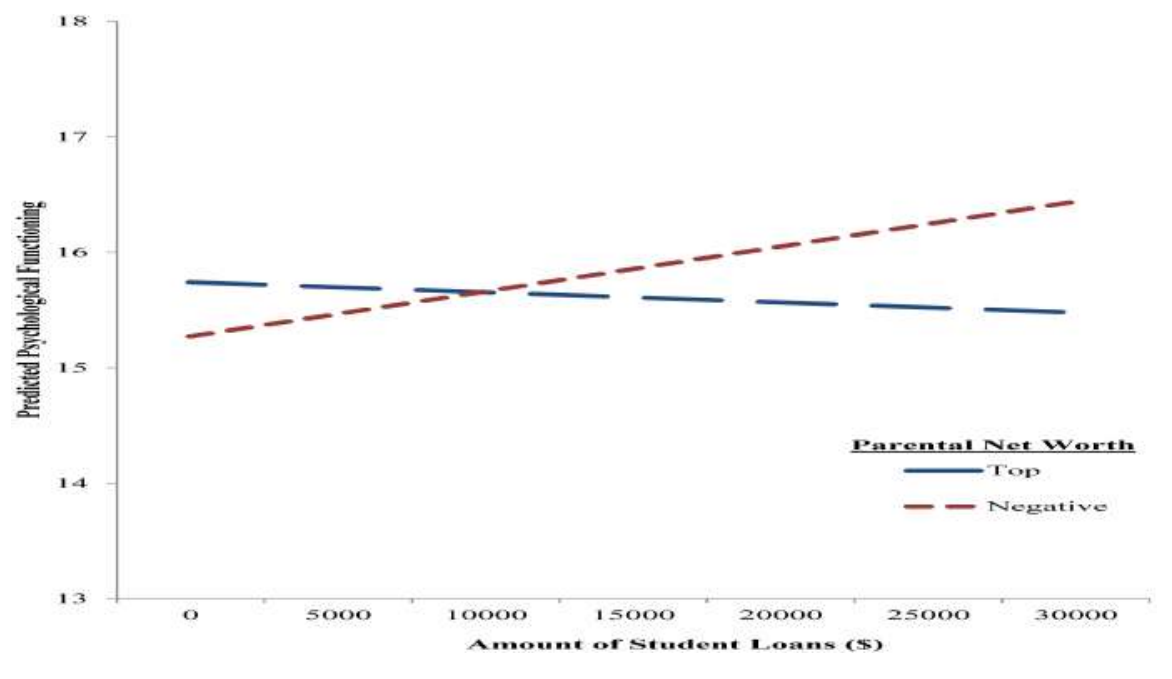

International Journal of Developmental and Educational Psychology 
Asimismo indicar que, en países anglosajones como Reino Unido, y en especial USA, donde el coste de las universidades requiere la petición de créditos o becas que dependen del desempeño que tenga el alumno, este criterio juega un papel destacado en la salud mental de los estudiantes. Se ha establecido relación entre la cantidad de dinero que se pidió prestado en un crédito universitario y el funcionamiento psicológico de dichos individuos entre los 25 y 31 años, observando que había una correlación inversa entre la cantidad de dinero pedida y el desempeño psicológico. (Walsemann et al., 2015)

Como se aprecia la situación de dificultad emocional, de riesgo per se, es más prevalente de lo que se podría pensar en un primer momento, de ahí la importancia de una respuesta clara a nivel institucional dentro de la propia universidad, venciendo el miedo a la discriminación y a las repercusiones laborales posteriores. (Macaskill, 2013) La intervención más ofrecida por la mayoría de las instituciones es el "counselling", y aunque tiene buenos resultados, debido a la cantidad de alumnado que puede demandarlo y a los recursos limitados con los que cuentan las instituciones académicas resulta todavía insuficiente para dar respuesta a estas nuevas necesidades desarrolladas por el alumnado en pleno siglo XXI. (Holm-Hadulla \& Koutsoukou-Argyraki, 2015)

Estudios realizados como, por ejemplo, los de Broglia et al., en 2018, confirman la necesidad de analizar, debido a la gran demanda de los estudiantes, este tipo de servicios y de conocer cuál es su verdadera efectividad y lo que es más interesante, cómo de útiles creen los estudiantes que les resultan para el acompañamiento y el tratamiento de sus problemas.

\section{MÉTODO}

Se trata de un estudio observacional descriptivo donde se ha realizado una revisión sistemática en bases de datos bibliográficas (Pub-Med/MEDLINE, Scielo, SCOPUS, ISI Web of Knowledge, CRD, IME, IBECS) y manualmente a través de buscadores como Google Académico en revistas y organismos públicos con documentos que tratan la idea-fuerza seleccionada. Los estudios científicos y las revisiones sobre el tema a investigar se han valorado a partir los últimos 20 años, tanto en la literatura científica de habla hispana como de habla anglosajona.

Para seleccionar los artículos se han empleado unos criterios de inclusión y exclusión en el que se ha tenido en cuenta para seleccionar los artículos cuyas palabras clave incluían como descriptores del MESH "salud mental", "mental health", "estudiantes universitarios" "college students", "university students". Además se han elegido aquellos artículos según su temporalidad, ciñéndonos a los últimos 15 años en el estudio de la investigación. Así mismo con respecto a la extracción de datos, se han localizado unos 50 artículos de los que se han seleccionado 10 que cumplían los criterios de inclusión. Dichos estudios han evaluado varios puntos relevantes; el impacto de la salud mental en los estudiantes universitarios de distintas partes del globo, así como la salud mental en ciertos grupos de estudiantes de carreras de las rama sanitaria, y su comparación con otros grados, amén de otras variantes que caracterizan cada estudio de manera individual, como la valoración de la inteligencia emocional 0 de las variables sociodemográficas. Asimismo se han escogido estudios que empleaban algún tipo de herramienta cualitativa como test o escalas para valorar la incidencia de las enfermedades mentales, dando prioridad a aquellos que tenían menor antigüedad.

\section{RESULTADO Y DISCUSIÓN}

De las fuentes primarias identificamos un primer resultado: todas ellas han realizado un estudio sobre estudiantes y han concluido aunque de manera desigual que, no tienen mayor incidencia de enfermedades mentales que el resto de la población. Sin embargo se ha visibilizado que los síntomas psiquiátricos están más presentes en los estudiantes de las ramas sociosanitarias, y que en función de las distintos Grados universitarios parece que las enfermedades mentales más prevalentes cambian pero todas se encuentran dentro de la esfera ansiosodepresiva. Hay múltiples factores que pueden influir en su aparición aunque está modulado por una serie de situaciones consideradas como "protectoras" para el alumnado universitario, esto es, tener una adecuada red de apoyo familiar o tener un buen sistema de apoyo institucional dentro de la universidad (unidades o servicios de atención al estudiante); del mismo modo, encontramos situaciones de riesgo especialmente frecuente en el ámbi- 
to americano como los préstamos universitarios o las mayores exigencias según las carreras y las universidades; esta última variable también parece que cobra una importancia mayor, donde los alumnos indican que las exigencias académicas o el poco tiempo para el estudio así como la sobrecarga de actividades son factores extrínsecos de problemas mentales.

Aunque la relación entre el desempeño académico y el rendimiento psicológico, según Bruffaerts et al. (2018), todavía no está comprendida adecuadamente, se han desarrollado diversos estudios con el objetivo de valorar hasta qué punto los problemas mentales están asociados con el funcionamiento académico; aproximadamente uno de cada tres nuevos alumnos reportan problemas de salud mental en el pasado año, estando éstos relacionados con una reducción del funcionamiento académico, además se encontró que los departamentos que reportaban una menor productividad presentaban una mayor incidencia de enfermedades mentales.

Se ha observado, en ese sentido, que muchas instituciones universitarias han valorado las características previas de los estudiantes que han escogido esas carreras como su ambiente familiar o si padecían alguna patología previa antes de entrar en la carrera.

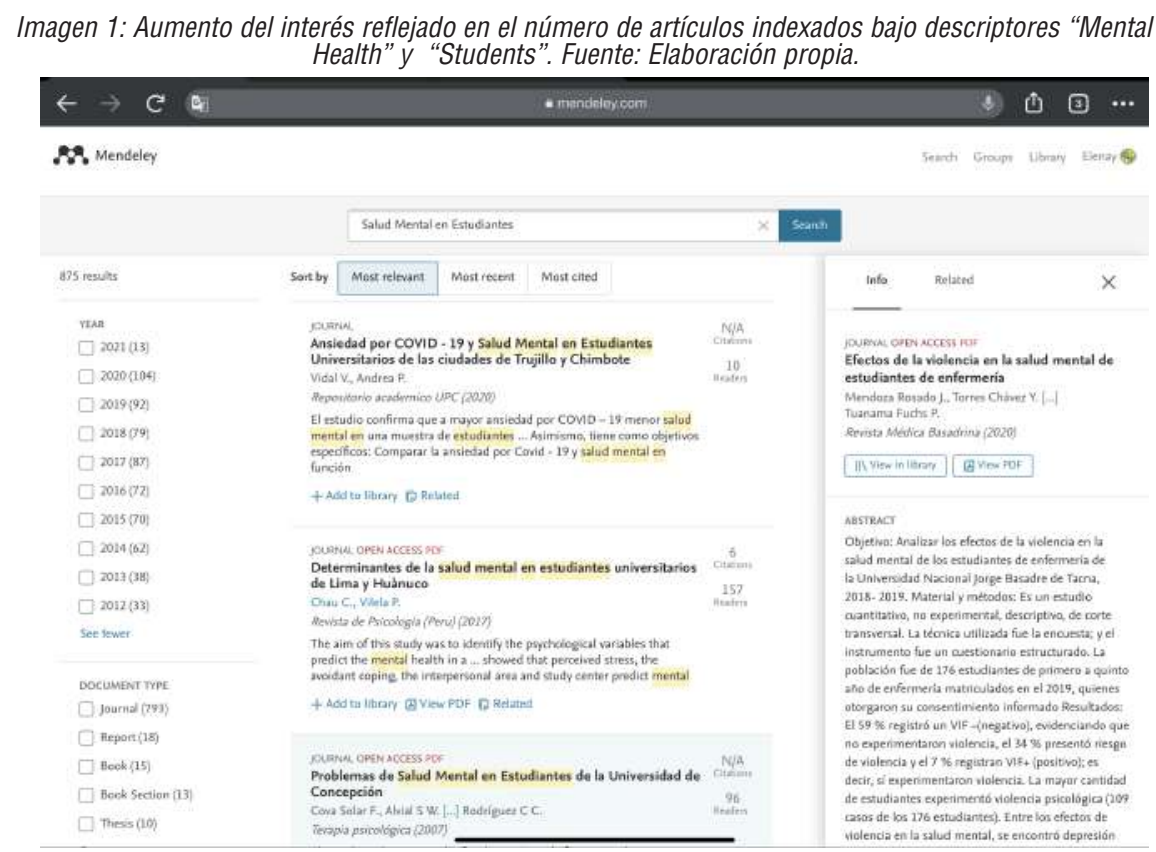

Analizando el material obtenido se aprecia que desde el 2016 se ha observado un aumento exponencial en el interés que ha despertado la salud mental de los estudiantes universitarios, especialmente en aquellos que cursan sus estudios en la rama sociosanitaria, en el ámbito de la investigación. Esta situación se ha visto incrementada por la pandemia, donde se ha subrayado la sin par relevancia no solo de la salud mental "sensu estricto", sino como un elemento añadido en relación directa con la productividad académica del alumnado de las ramas sociosanitarias.

Si la situación de la salud mental en estudiantes universitarios ha empezado a ser un tema de interés general en el ámbito psicológico, está claro que debido a la presencia de la Covid-19 y de todos los cambios que ésta ha requerido globalmente, podemos observar que se ha puesto en valor, todavía más, la necesidad de atender a este colectivo. (Morote-Jayacc et al., 2020) 
Tabla 1: Comparativa de los diferentes estudios elegidos para la revisión bibliográfica. Parte 1

\begin{tabular}{|c|c|c|c|c|c|}
\hline Título & $\begin{array}{l}\text { “The mental } \\
\text { health of } \\
\text { university } \\
\text { students in } \\
\text { the United } \\
\text { Kingdom" }\end{array}$ & $\begin{array}{c}\text { “Problemas } \\
\text { de Salud } \\
\text { Mental en } \\
\text { Estudiantes } \\
\text { de la } \\
\text { Universidad } \\
\text { de } \\
\text { Concepción" }\end{array}$ & $\begin{array}{c}\text { “Salud mental } \\
\text { en estudiantes } \\
\text { de odontología } \\
\text { de una } \\
\text { Universidad } \\
\text { Pública de } \\
\text { Medellín } \\
\text { (Colombia) y } \\
\text { sus factores } \\
\text { relacionados" }\end{array}$ & $\begin{array}{c}\text { “Problemas de } \\
\text { salud mental } \\
\text { en estudiantes } \\
\text { de una } \\
\text { universidad } \\
\text { regional } \\
\text { chilena" }\end{array}$ & $\begin{array}{c}\text { “Inteligencia } \\
\text { emocional } \\
\text { asociada a } \\
\text { salud mental } \\
\text { en estudiantes } \\
\text { universitarios } \\
\text { de la carrera } \\
\text { de Psicología” }\end{array}$ \\
\hline $\begin{array}{c}\text { Autor } \\
\text { Revista } \\
\text { Año }\end{array}$ & $\begin{array}{l}\text { A. } \\
\text { Mackaskill. } \\
\text { “Journal of } \\
\text { Mental } \\
\text { Health" } \\
2018\end{array}$ & $\begin{array}{l}\text { Cova Solar F. } \\
\text { Alvial S W, } \\
\text { Aro D. M. } \\
\text { "Terapia } \\
\text { Psicológica" } \\
2007\end{array}$ & $\begin{array}{l}\text { Álvarez Rúa, } \\
\text { Laura J. } \\
\text { Et al. } \\
\text { "Revista } \\
\text { Nacional de } \\
\text { Odontología" } \\
2019\end{array}$ & $\begin{array}{l}\text { Antúnez } \\
\text { Sanhueza, } \\
\text { Zayra } \\
\text { Vinet, Eugenia } \\
\text { V. } \\
\text { "Revista } \\
\text { Médica de } \\
\text { Chile" } \\
2013\end{array}$ & $\begin{array}{l}\text { Duarte Ortíz, } \\
\text { F. } \\
\text { "Innovare:Revi } \\
\text { sta de Ciencia } \\
\text { y Tecnología" } \\
2019\end{array}$ \\
\hline Objetiv & $\begin{array}{l}\text { Este estudio } \\
\text { valora los } \\
\text { niveles de } \\
\text { enfermedad } \\
\text { mental en } \\
\text { estudiantes } \\
\text { universitarios } \\
\text { para examinar } \\
\text { si su } \\
\text { participación } \\
\text { en la } \\
\text { educación a } \\
\text { estos niveles } \\
\text { ha resultado } \\
\text { en un } \\
\text { aumento de } \\
\text { las mismas. }\end{array}$ & $\begin{array}{l}\text { Estudio } \\
\text { mediante } \\
\text { muestreo por } \\
\text { conglomerad } \\
\text { o valorando } \\
\text { la presencia } \\
\text { de síndrome } \\
\text { ansioso, } \\
\text { depresivo o } \\
\text { sintomatologí } \\
\text { a asociada al } \\
\text { estrés } \\
\text { académico. }\end{array}$ & $\begin{array}{l}\text { Analizar la } \\
\text { prevalencia de } \\
\text { signos y } \\
\text { síntomas } \\
\text { sugerentes de } \\
\text { estrés, } \\
\text { depresión } \\
\text { /ansiedad y } \\
\text { mala salud } \\
\text { mental y sus } \\
\text { factores socio- } \\
\text { demográficos } \\
\text { relacionados en } \\
\text { estudiantes de } \\
\text { pregrado. }\end{array}$ & $\begin{array}{l}\text { Determinar la } \\
\text { frecuencia de } \\
\text { enfermedades } \\
\text { mentales, } \\
\text { problemas de } \\
\text { salud, en } \\
\text { asociación con } \\
\text { otras variables } \\
\text { relevantes entre } \\
\text { los estudiantes } \\
\text { de la } \\
\text { Universidad de } \\
\text { Chile. }\end{array}$ & $\begin{array}{l}\text { El objetivo de } \\
\text { esta } \\
\text { investigación } \\
\text { es presentar los } \\
\text { resultados de } \\
\text { estudio de la } \\
\text { inteligencia } \\
\text { emocional y su } \\
\text { relación con la } \\
\text { salud mental }\end{array}$ \\
\hline Método & $\begin{array}{l}\text { Se trata de un } \\
\text { estudio } \\
\text { observacional } \\
\text { cruzado de } \\
\text { tipo } \\
\text { descriptivo } \\
\text { donde se han } \\
\text { implementad } \\
\text { o un }\end{array}$ & $\begin{array}{l}\text { Se eligió } \\
\text { aleatoriament } \\
\text { e del total de } \\
\text { Facultades de } \\
\text { la } \\
\text { Universidad } \\
\text { de } \\
\text { Concepción, } \\
\text { donde el }\end{array}$ & $\begin{array}{l}\text { Se trata de un } \\
\text { estudio } \\
\text { transversal } \\
\text { mediante una } \\
\text { encuesta } \\
\text { midiendo } \\
\text { diversas } \\
\text { variables como } \\
\text { salud mental }\end{array}$ & $\begin{array}{l}\text { Una muestra de } \\
484 \text { estudiantes } \\
\text { de la } \\
\text { Universidad de } \\
\text { Chile, de edad } \\
\text { comprendida } \\
\text { entre } 18-28 \\
\text { años. }\end{array}$ & $\begin{array}{l}\text { Se empleó una } \\
\text { metodología de } \\
\text { tipo } \\
\text { cuantitativo, } \\
\text { transversal, } \\
\text { con un alcance } \\
\text { descriptivo } \\
\text { correlacional y } \\
\text { diseño no }\end{array}$ \\
\hline
\end{tabular}




\begin{tabular}{|c|c|c|c|c|c|}
\hline & $\begin{array}{l}\text { cuestionario } \\
\text { sobre salud a } \\
\text { los } \\
\text { estudiantes } \\
\text { universitarios } \\
\text { en distintos } \\
\text { cursos. }\end{array}$ & $\begin{array}{l}\text { número total } \\
\text { de alumnos } \\
\text { es } 15.309 \text {. }\end{array}$ & $\begin{array}{l}\text { (GHQ-12) o la } \\
\text { depresión } \\
\text { ansiedad (test } \\
\text { de Zung). }\end{array}$ & & $\begin{array}{l}\text { experimental. } \\
\text { Recolectó la } \\
\text { información } \\
\text { con dos } \\
\text { pruebas: Trait } \\
\text { Meta Mood } \\
\text { Scale TMMS- } \\
24 \text { y la escala } \\
\text { de Salud } \\
\text { General de } \\
\text { Goldberg } \\
\text { GHQ-28, }\end{array}$ \\
\hline $\begin{array}{c}\text { Sujetos de } \\
\text { Origen }\end{array}$ & $\begin{array}{l}\text { La muestra de } \\
\text { estudiantes } \\
\text { fue de } 1197 \\
\text { completaron } \\
\text { el “General } \\
\text { Health } \\
\text { Questionnaire } \\
-28 \text { " en el } \\
\text { primer día de } \\
\text { universidad, a } \\
\text { mediados del } \\
\text { primer año o } \\
\text { en su segundo } \\
\text { o tercer año } \\
\text { como } \\
\text { estudiantes. }\end{array}$ & & $\begin{array}{l}\text { El estudio se ha } \\
\text { realizado sobre } \\
205 \\
\text { estudiantes, } \\
104 \text { de ellos } \\
\text { mujeres de } \\
\text { pregrado de la } \\
\text { Facultad de } \\
\text { Odontología de } \\
\text { la Universidad } \\
\text { de Antioquía. }\end{array}$ & $\begin{array}{l}\text { La batería de } \\
\text { test utilizados } \\
\text { para detectar } \\
\text { problemas de } \\
\text { salud mental. } \\
\text { Algunos de } \\
\text { ellos eran: } \\
\text { "Derogates } \\
\text { SCL-90-R", } \\
\text { "Beck Anxiety } \\
\text { Inventory } \\
\text { (BAI)", "Beck } \\
\text { Depression } \\
\text { Inventory } \\
\text { (BDI-II"), } \\
\text { "Depression, } \\
\text { Anxiety and } \\
\text { Stress Scales } \\
\text { (DASS-21)"y } \\
\text { un Questionario } \\
\text { Socio- } \\
\text { Demográfico. }\end{array}$ & $\begin{array}{l}\text { El grupo de } \\
\text { estudio fue un } \\
\text { grupo de } 129 \\
\text { estudiantes que } \\
\text { forman el } \\
\text { universo del } \\
\text { primer ingreso } \\
\text { en el III } \\
\text { período } \\
\text { académico } \\
2014 .\end{array}$ \\
\hline Resultado & \begin{tabular}{|l|} 
Las tasas de \\
enfermedad \\
mental en \\
estudiantes \\
eran similares \\
a las \\
detectadas en \\
la población \\
general, pero \\
solo un 5,1\% \\
se \\
encontraban \\
actualmente \\
en
\end{tabular} & $\begin{array}{l}\text { Se encontró } \\
\text { un } 23,4 \% \text { de } \\
\text { síndrome } \\
\text { ansioso, un } \\
16,4 \% \text { de } \\
\text { síndrome } \\
\text { depresivo. La } \\
\text { presencia de } \\
\text { sintomatologí } \\
\text { a ligada al } \\
\text { estrés } \\
\text { académico } \\
\text { fue una de las } \\
\text { problemáticas }\end{array}$ & $\begin{array}{l}\text { La asociación } \\
\text { fue } \\
\text { estadísticament } \\
\text { e significativa } \\
\text { en los hombres } \\
\text { que reportaron } \\
\text { depresión/ } \\
\text { ansiedad y } \\
\text { estrés y apoyo } \\
\text { social bajo (OR } \\
\text { 20,94; IC95\% } \\
2,13-205,81 \text { y } \\
\text { OR 5,04; } \\
\text { IC95\% 1,01- }\end{array}$ & $\begin{array}{l}\text { Un porcentaje } \\
\text { relevante de la } \\
\text { muestra } \\
\text { presentó } \\
\text { niveles } \\
\text { elevados de } \\
\text { síntomas } \\
\text { determinados } \\
\text { por los puntos } \\
\text { de corte de los } \\
\text { inventores de } \\
\text { Beck. } \\
\text { El grupo } \\
\text { sintomático }\end{array}$ & $\begin{array}{l}\text { Los resultados } \\
\text { indican que } \\
\text { niveles altos de } \\
\text { inteligencia } \\
\text { emocional } \\
\text { están } \\
\text { significativame } \\
\text { nte } \\
\text { relacionados } \\
\text { con una buena } \\
\text { salud mental } \\
\text { mientras que } \\
\text { niveles bajos } \\
\text { de esta se }\end{array}$ \\
\hline
\end{tabular}




\begin{tabular}{|c|c|c|c|c|}
\hline $\begin{array}{l}\text { tratamiento. } \\
\text { En el segundo } \\
\text { año de } \\
\text { estudio es } \\
\text { cuando mayor } \\
\text { se reportaron } \\
\text { síntomas } \\
\text { psiquiátricos. }\end{array}$ & $\begin{array}{l}\text { observadas } \\
\text { más } \\
\text { frecuentes. } \\
\text { También se } \\
\text { apreciaron } \\
\text { cifras } \\
\text { elevadas } \\
\text { relacionadas } \\
\text { con el deseo } \\
\text { de muerte e } \\
\text { ideación } \\
\text { suicida. }\end{array}$ & $\begin{array}{l}25,02 \\
\text { respectivament } \\
\text { e). También se } \\
\text { reportó mayor } \\
\text { riesgo en los } \\
\text { hombres que } \\
\text { refieren } \\
\text { depresión/ } \\
\text { ansiedad y con } \\
\text { familias } \\
\text { extensas- } \\
\text { ensambladas } \\
\text { (OR: 8,82; } \\
\text { IC95\% 1,29- } \\
60,17) \text {. }\end{array}$ & $\begin{array}{l}\text { demostró } \\
\text { niveles } \\
\text { elevados de } \\
\text { estrés y estaba } \\
\text { compuesto } \\
\text { principalmente } \\
\text { por estudiantes } \\
\text { de nuevo } \\
\text { ingreso y } \\
\text { mujeres. Hubo } \\
\text { asociaciones } \\
\text { significativas } \\
\text { entre los } \\
\text { síntomas y las } \\
\text { variables socio- } \\
\text { demográficas } \\
\text { como vivir en } \\
\text { áreas rurales, la } \\
\text { etnia, el estatus } \\
\text { socioeconómic } \\
\text { o, la necesidad } \\
\text { de apoyo } \\
\text { psicológico y } \\
\text { psiquiátrico, } \\
\text { con un menor } \\
\text { número de } \\
\text { actividades } \\
\text { extracurriculare } \\
\text { s. }\end{array}$ & $\begin{array}{l}\text { asocian a una } \\
\text { pobre salud } \\
\text { mental e } \\
\text { incluso se } \\
\text { entienden } \\
\text { como } \\
\text { indicadores de } \\
\text { precariedad } \\
\text { emocional, } \\
\text { considerada } \\
\text { como caso } \\
\text { psicológico }\end{array}$ \\
\hline
\end{tabular}


Tabla 1: Comparativa de los diferentes estudios elegidos para la revisión bibliográfica. Parte 2

\begin{tabular}{|c|c|c|c|c|c|}
\hline Título & $\begin{array}{c}\text { “Estrés y } \\
\text { Salud } \\
\text { Mental en } \\
\text { Estudiantes } \\
\text { de Medicina: } \\
\text { Relación con } \\
\text { Afrontamient } \\
\text { oy } \\
\text { Actividades } \\
\text { Extracurricu } \\
\text { lares" }\end{array}$ & $\begin{array}{l}\text { “Determinante } \\
\text { s de la salud } \\
\text { mental en } \\
\text { estudiantes } \\
\text { universitarios } \\
\text { de Lima y } \\
\text { Huànuco” }\end{array}$ & $\begin{array}{c}\text { "Assessing } \\
\text { student mental } \\
\text { health at the } \\
\text { Vanderbilt } \\
\text { University } \\
\text { School of } \\
\text { Medicine" }\end{array}$ & $\begin{array}{c}\text { “Health } \\
\text { assessment of } \\
\text { French } \\
\text { university } \\
\text { students and } \\
\text { risk factors } \\
\text { associated with } \\
\text { mental health } \\
\text { disorders" }\end{array}$ & $\begin{array}{c}\text { “Depresión en } \\
\text { estudiantes } \\
\text { universitarios y } \\
\text { su asociación } \\
\text { con el estrés } \\
\text { académico" }\end{array}$ \\
\hline $\begin{array}{c}\text { Autor } \\
\text { Revista } \\
\text { Año }\end{array}$ & $\begin{array}{l}\text { Lemos M. } \\
\text { Henaos M. } \\
\text { López D. } \\
\text { “iMedPub } \\
\text { Journals” } \\
2018\end{array}$ & $\begin{array}{l}\text { Chau C. } \\
\text { Vilela P. } \\
\text { "Revista de } \\
\text { Psicología } \\
\text { (Perú)" } \\
2017\end{array}$ & $\begin{array}{l}\text { Ghodasara, } \\
\text { Sweta L. } \\
\text { Davidson, } \\
\text { Mario A. } \\
\text { Reich, Michael } \\
\text { S. } \\
\text { Savoie, Corliss } \\
\text { V. } \\
\text { Rodgers, Scott } \\
\text { M. } \\
\text { "Academic } \\
\text { Medicine" } \\
2011\end{array}$ & $\begin{array}{l}\text { Tran, A. } \\
\text { Tran, L. } \\
\text { Geghre, N. } \\
\text { "PLoS ONE" } \\
2017\end{array}$ & $\begin{array}{l}\text { Vélez, L. } \\
\text { Gutierrez, J. } \\
\text { et al. } \\
\text { "CES } \\
\text { Medicina" } \\
2010 .\end{array}$ \\
\hline Objetivo & $\begin{array}{l}\text { Se caracteriza } \\
\text { los niveles de } \\
\text { estrés, salud } \\
\text { mental y su } \\
\text { asociación } \\
\text { con variables } \\
\text { psicológicas, } \\
\text { fisiológicas y } \\
\text { de educación } \\
\text { en } \\
\text { estudiantes } \\
\text { de medicina } \\
\text { colombianos }\end{array}$ & $\begin{array}{l}\text { El objetivo de } \\
\text { este estudio es } \\
\text { identificar las } \\
\text { variables } \\
\text { psicológicas } \\
\text { para predecir la } \\
\text { salud mental. }\end{array}$ & $\begin{array}{l}\text { El objetivo de } \\
\text { este estudio es } \\
\text { determinar la } \\
\text { prevalencia de } \\
\text { las cuatro } \\
\text { principales } \\
\text { categorías de } \\
\text { enfermedad } \\
\text { mental entre } \\
\text { los estudiantes } \\
\text { de medicina } \\
\text { para examinar } \\
\text { las } \\
\text { asociaciones } \\
\text { entre estas } \\
\text { enfermedades } \\
\text { y el rango de } \\
\text { variables } \\
\text { demográficas. }\end{array}$ & $\begin{array}{l}\text { El objetivo de } \\
\text { este estudio es } \\
\text { evaluar la } \\
\text { salud y los } \\
\text { estilos de vida } \\
\text { en estudiantes } \\
\text { de grado, así } \\
\text { como evaluar } \\
\text { los posibles } \\
\text { factores de } \\
\text { riesgo } \\
\text { asociados con } \\
\text { los síntomas } \\
\text { psiquiátricos. }\end{array}$ & $\begin{array}{l}\text { Este estudio } \\
\text { tenía como } \\
\text { objetivo } \\
\text { principal } \\
\text { investigar sobre } \\
\text { los múltiples } \\
\text { factores que } \\
\text { pueden afectar a } \\
\text { la salud mental } \\
\text { de los } \\
\text { estudiantes } \\
\text { universitarios y } \\
\text { su posible } \\
\text { relación con la } \\
\text { presencia de } \\
\text { depresión. }\end{array}$ \\
\hline
\end{tabular}




\begin{tabular}{|c|c|c|c|c|c|}
\hline Método & $\begin{array}{l}\text { Estudio } \\
\text { transversal } \\
\text { mediante un } \\
\text { Cuestionario } \\
\text { de estrés } \\
\text { percibido } \\
\text { (PSS14), } \\
\text { Cuestionario } \\
\text { de salud } \\
\text { (PHQ-9) para } \\
\text { depresión, } \\
\text { cuestionario } \\
\text { de ansiedad } \\
\text { generalizada } \\
\text { (GAD 7), } \\
\text { Cuestionario } \\
\text { de Apoyo } \\
\text { Social (MOS- } \\
\text { SSS) y } \\
\text { cuestionario } \\
\text { de estrategias } \\
\text { de } \\
\text { afrontamient } \\
\text { o modificado; } \\
\text { así como } \\
\text { medidas } \\
\text { fisiológicas y } \\
\text { un registro de } \\
\text { actividades } \\
\text { extracurricula } \\
\text { res. }\end{array}$ & $\begin{array}{l}\text { Para esto se } \\
\text { han utilizado } \\
\text { las siguientes } \\
\text { escalas } \\
\text { "General Self- } \\
\text { efficacy", } \\
\text { "Coping } \\
\text { Estimation } \\
\text { Inventory } \\
\text { (COPE 60)", } \\
\text { "Academic } \\
\text { Experiences } \\
\text { Questionnaire } \\
\text { (QVA-r)", } \\
\text { "Perceived } \\
\text { Stress Scale } \\
\text { (PSS)"y } \\
\text { "SF36 } \\
\text { questionnaire". }\end{array}$ & $\begin{array}{l}\text { Los estudiantes } \\
\text { completaron } \\
\text { un cuestionario } \\
\text { anónimo } \\
\text { valorando la } \\
\text { prevalencia de } \\
\text { la depresión, } \\
\text { ansiedad, } \\
\text { trastornos de la } \\
\text { conducta } \\
\text { alimentaria y } \\
\text { problemas de } \\
\text { consumo de } \\
\text { drogas. } \\
\text { Además los } \\
\text { autores } \\
\text { obtienen } \\
\text { adicionalmente } \\
\text { una } \\
\text { información } \\
\text { demográfica } \\
\text { para investigar } \\
\text { las variaciones } \\
\text { de las tasas de } \\
\text { la enfermedad } \\
\text { basada en las } \\
\text { diferencias } \\
\text { interindividual } \\
\text { es }\end{array}$ & $\begin{array}{l}\text { Los datos } \\
\text { fueron } \\
\text { recopilados } \\
\text { prospectivame } \\
\text { nte mediante la } \\
\text { base de datos } \\
\text { CALCIUM. } \\
\text { (Consultations } \\
\text { Assistés par } \\
\text { Logiciel pour } \\
\text { les Centres } \\
\text { Inter- } \\
\text { Universitaire } \\
\text { de Médecine) y } \\
\text { se incluyó la } \\
\text { información } \\
\text { sobre el estilo } \\
\text { de vida de los } \\
\text { estudiantes (las } \\
\text { condiciones de } \\
\text { vida, la dieta, } \\
\text { la actividad } \\
\text { fisica o el uso } \\
\text { recreacional } \\
\text { de drogas). La } \\
\text { prevalencia de } \\
\text { síntomas } \\
\text { psiquiátricos } \\
\text { relacionados } \\
\text { con la } \\
\text { depresión, la } \\
\text { ansiedad y los } \\
\text { ataques de } \\
\text { pánicos fue } \\
\text { evaluada, así } \\
\text { como se } \\
\text { analizaron los } \\
\text { factores de } \\
\text { riesgo } \\
\text { mediante una } \\
\text { regresión } \\
\text { logística. } \\
\text { a }\end{array}$ & $\begin{array}{l}\text { Se aplicó un } \\
\text { instrumento } \\
\text { auto- } \\
\text { administrado a } \\
\text { todos los } \\
\text { estudiantes } \\
\text { matriculados en } \\
\text { los programas } \\
\text { de pregrado de } \\
\text { la Universidad } \\
\text { CES que } \\
\text { permitió } \\
\text { establecer la } \\
\text { prevalencia de } \\
\text { depresión y el } \\
\text { Chi2 de } \\
\text { tendencia para } \\
\text { ver el } \\
\text { comportamiento } \\
\text { de la relación } \\
\text { entre el nivel de } \\
\text { estrés y la } \\
\text { depresión. El } \\
\text { análisis } \\
\text { estadístico se } \\
\text { realizó con el } \\
\text { programa } \\
\text { SPSS } ® .\end{array}$ \\
\hline $\begin{array}{l}\text { Sujetos } \\
\text { de } \\
\text { Origen }\end{array}$ & $\begin{array}{l}\text { El grupo de } \\
\text { estudio fue } \\
\text { un grupo de } \\
217 \\
\text { estudiantes } \\
\text { seleccionados }\end{array}$ & $\begin{array}{l}\text { La muestra ha } \\
\text { sido de } 1.024 \\
\text { estudiantes de } \\
\text { Lima y } \\
\text { Huànuco. }\end{array}$ & $\begin{array}{l}\text { Los autores } \\
\text { han incluido a } \\
330 \text { estudiantes } \\
\text { de Medicina de } \\
1^{\circ}, 2^{\circ} \text { y } 3^{\circ} \\
\text { curso de la }\end{array}$ & \begin{tabular}{|l} 
Entre \\
Septiembre de \\
2012 y Junio \\
2013, se han \\
incluido todos \\
los estudiantes
\end{tabular} & $\begin{array}{l}\text { La población } \\
\text { estudiada estuvo } \\
\text { conformada por } \\
1344 \text { estudiantes } \\
\text { entre los } 16 \text { y } \\
\text { los } 37 \text { años, con }\end{array}$ \\
\hline
\end{tabular}




\begin{tabular}{|c|c|c|c|c|c|}
\hline & $\begin{array}{l}\text { aleatoriament } \\
\text { e } \\
\text { estratificando } \\
\text { s según el } \\
\text { ciclo } \\
\text { académico. }\end{array}$ & & $\begin{array}{l}\text { Universidad de } \\
\text { Vanderbilt } \\
\text { durante el } \\
\text { invierno de } \\
2008-2009 .\end{array}$ & $\begin{array}{l}\text { universitarios } \\
\text { que acudieron } \\
\text { a una revisión } \\
\text { obligatoria en } \\
\text { el servicio } \\
\text { médico de la } \\
\text { Universidad de } \\
\text { Niza, durante } \\
\text { la cual se les } \\
\text { realizó una } \\
\text { anamnesis de } \\
\text { potenciales } \\
\text { enfermedades. }\end{array}$ & $\begin{array}{l}\text { una edad } \\
\text { promedio y } \\
\text { desviación } \\
\text { estándar de } 20,4 \\
\pm 2,6 \text { años. El } \\
68,7 \% \text { de la } \\
\text { población es de } \\
\text { género femenino }\end{array}$ \\
\hline $\begin{array}{c}\text { Resultad } \\
\text { o }\end{array}$ & $\begin{array}{l}\text { Se } \\
\text { presentaron } \\
\text { niveles altos } \\
\text { de estrés } \\
(64 \%), \\
\text { depresión } \\
(56.2 \%) \text { y } \\
\text { ansiedad } \\
(48.3 \%) . \\
\text { Mediante una } \\
\text { regresión } \\
\text { logística } \\
\text { bivariada se } \\
\text { encontró que } \\
\text { ser mujer } \\
\text { (OR 2.64, } \\
\text { I.C. 95\% } \\
1.29-5.39), \\
\text { estar en el } \\
\text { ciclo básico } \\
\text { (OR 0.38, } \\
\text { I.C. 95\% } \\
0.19-0.78), \\
\text { no realizar } \\
\text { actividades } \\
\text { extra- } \\
\text { curriculares } \\
\text { (OR 2.14, } \\
\text { I.C. 95\% } \\
\text { 1.05-4.36), } \\
\text { bajas } \\
\text { puntuaciones } \\
\text { en solución } \\
\text { de problemas } \\
\text { (OR 0.93, }\end{array}$ & $\begin{array}{l}\text { Los resultados } \\
\text { mostraron } \\
\text { como la } \\
\text { percepción del } \\
\text { estrés, la falta } \\
\text { de mecanismos } \\
\text { de respuesta y } \\
\text { el área } \\
\text { interpersonal } \\
\text { eran } \\
\text { predictores de } \\
\text { la salud } \\
\text { mental. El } \\
\text { análisis } \\
\text { realizado } \\
\text { reveló que el } \\
\text { estrés en los } \\
\text { estudiantes está } \\
\text { relacionado } \\
\text { con la falta de } \\
\text { mecanismos de } \\
\text { respuesta y con } \\
\text { su salud mental } \\
\text { general. }\end{array}$ & $\begin{array}{l}\text { La mayoría de } \\
\text { los estudiantes } \\
\text { (301; response } \\
\text { rate: } 91.2 \%) \\
\text { completaron la } \\
\text { encuesta. Los } \\
\text { autores } \\
\text { encontraron } \\
\text { que la } \\
\text { depresión y la } \\
\text { ansiedad eran } \\
\text { más prevalente } \\
\text { en los } \\
\text { estudiantes de } \\
\text { Medicina que } \\
\text { en los } \\
\text { universitarios } \\
\text { de otros grados } \\
\text { no médicos. } \\
\text { Los autores } \\
\text { encontraron } \\
\text { que } 37 \text { (12\%) } \\
\text { de los } \\
\text { estudiantes } \\
\text { rozaban el } \\
\text { alcoholismo } \\
\text { for y } 3(1 \%) \\
\text { tenía ya } \\
\text { problemas con } \\
\text { la bebida, } 1 \\
(0.3 \%) \text { tenía un } \\
\text { trastorno por } \\
\text { consumo de } \\
\text { sustancias, } 3 \\
(1 \%) \text { tenían }\end{array}$ & $\begin{array}{l}\text { Un total de } \\
4,184 \\
\text { estudiantes } \\
\text { universitarios } \\
\text { fueron } \\
\text { incluidos. La } \\
\text { prevalencia de } \\
\text { la depresión, } \\
\text { ansiedad y } \\
\text { ataques de } \\
\text { pánico fue de } \\
12.6 \%, 7.6 \% \text { y } \\
1.0 \% \text {, } \\
\text { respectivament } \\
\text { e. Durante los } \\
30 \text { días previos } \\
\text { a la evaluación, } \\
0.6 \% \text { de los } \\
\text { estudiantes } \\
\text { bebieron } \\
\text { regularmente } \\
\text { alcohol. Otro, } \\
6.3 \% \text { eran } \\
\text { fumadores de } \\
\text { tabaco y } 10.0 \% \\
\text { fumaban } \\
\text { marihuana. Así } \\
\text { mismo se } \\
\text { observaron una } \\
\text { serie de } \\
\text { asociaciones, } \\
\text { como la de } \\
\text { tener } \\
\text { dificultades } \\
\text { financieras y }\end{array}$ & $\begin{array}{l}\text { La edad } \\
\text { promedio de los } \\
\text { estudiantes fue } \\
\text { de } 20,4 \pm 2,6 \\
\text { años. El } 68,7 \% \\
\text { de la población } \\
\text { es de género } \\
\text { femenino. La } \\
\text { prevalencia para } \\
\text { depresión } \\
\text { encontrada fue } \\
\text { del } 47,2 \% \text { y la } \\
\text { relación de ésta } \\
\text { con la severidad } \\
\text { del estrés } \\
\text { generado por los } \\
\text { estresores } \\
\text { académicos fue } \\
\text { estadísticamente } \\
\text { significativa }\end{array}$ \\
\hline
\end{tabular}




\begin{tabular}{|c|c|c|}
\hline $\begin{array}{l}\text { I.C. } 95 \% 0.89 \\
-0.93) \text {, altas } \\
\text { en reacción } \\
\text { agresiva (OR } \\
1.13 \text {, I.C. } \\
95 \% 1.05- \\
1.22) \text { y } \\
\text { expresión de } \\
\text { dificultad de } \\
\text { afrontamient } \\
\text { o (OR } 1.17 \text {, } \\
\text { I.C. } 95 \% \\
1.04-1.30) \\
\text { fueron } \\
\text { factores de } \\
\text { riesgo para el } \\
\text { estrés y } \\
\text { síntomas } \\
\text { emocionales. }\end{array}$ & $\begin{array}{l}\text { problemas de } \\
\text { la conducta } \\
\text { alimentaria. } \\
\text { También tener } \\
\text { una familia } \\
\text { con } \\
\text { antecedentes } \\
\text { de enfermedad } \\
\text { mental estaba } \\
\text { asociado a } \\
\text { mayor } \\
\text { incidencia de } \\
\text { depresión y } \\
\text { ansiedad. Así } \\
\text { mismo, se } \\
\text { observó una } \\
\text { asociación } \\
\text { entre el sexo } \\
\text { femenino y la } \\
\text { mayoría de los } \\
\text { trastornos. }\end{array}$ & $\begin{array}{l}\text { de aprendizaje } \\
\text { y la incidencia } \\
\text { de trastornos } \\
\text { psicológicos, } \\
\text { otra de ellas } \\
\text { fue que los } \\
\text { estudiantes que } \\
\text { no estaban } \\
\text { satisfechos con } \\
\text { sus } \\
\text { condiciones de } \\
\text { vida y aquellos } \\
\text { con conductas } \\
\text { alimentarias } \\
\text { poco } \\
\text { saludables } \\
\text { tenían más } \\
\text { riesgo de } \\
\text { depresión. Lo } \\
\text { mismo que } \\
\text { aquellas } \\
\text { mujeres que } \\
\text { vivían solas. } \\
\text { Por último, se } \\
\text { apreció una } \\
\text { mayor } \\
\text { probabilidad de } \\
\text { padecer un } \\
\text { trastorno } \\
\text { concomitante } \\
\text { teniendo ya } \\
\text { una patología } \\
\text { psicológica } \\
\text { previa. }\end{array}$ \\
\hline
\end{tabular}

\section{CONCLUSIONES}

El estudio de la salud mental en los estudiantes ha sido un tema recurrente en los últimos años. Es especialmente importante valorar que a estas edades la prevalencia de las enfermedades mentales se iguala a la de la población general, y aumenta en ciertos subgrupos, como los estudiantes de las carreras socio sanitarias. Se ha observado que en la mayoría de los autores revisados destaca el campo de la patología ansioso-depresiva, así como el estrés como principales problemas a la hora de abordar la salud mental en universitarios.

Así mismo se ha visto que el desempeño académico se encuentra relacionado de manera muy estrecha con la salud mental que puedan tener los estudiantes, además de otros factores que puedan influir en ella, como las características familiares o la petición de créditos o las exigencias curriculares de sus Grados sociosanitarios.

Se ha observado en esa dirección, que las mujeres, que son socio-demográficamente, el grupo más numeroso en estas titulaciones se encuentran más predispuestas a tener problemas relacionados con la esfera ansioso-depresiva, mientras que los hombres suelen tener más problemas con la gestión de los entornos productivos y el estrés académico. 
Dentro de las distintas carreras de la rama sociosanitaria la incidencia de las enfermedades también son distintas, destacando en frecuencia la ansiedad, seguida de la depresión en Medicina o Psicología, e invirtiéndose en Grados como el de Enfermería. En ese sentido, se han valorado la importancia de los factores estresores en esas formaciones, sobre todo en Medicina, donde los estudiantes han reflejado la dificultad para cumplir con las actividades académicas, es decir, se ha visibilizado la sobrecarga que presentan a lo largo de su larga capacitación. De estos, destacan que los estudiantes de los cursos iniciales son los que más presentan este tipo de patologías.

La respuesta de las instituciones ante las necesidades de los alumnos en este área es un punto importante a valorar, ya que la mayoría destaca la necesidad de tener una buena infraestructura académica que pueda servir como red de apoyo a los estudiantes así como un ambiente que favorezca la expresión de estas situaciones, evitando así el estigma que presentan este tipo patologías y la opacidad vigente a nivel general frente a los problemas de salud mental.

\section{REFERENCIAS BIBLIOGRÁFICAS}

Antúnez Sanhueza, Z., \& Vinet, E. V. (2013). Problemas de salud mental en estudiantes de una universidad regional chilena. Revista Medica de Chile, 141(2), 209-216. https://doi.org/10.4067/S003498872013000200010

Broglia, E., Millings, A., \& Barkham, M. (2018). Challenges to addressing student mental health in embedded counselling services: a survey of UK higher and further education institutions. British Journal of Guidance and Counselling, 46(4), 441-455. https://doi.org/10.1080/03069885.2017.1370695

Brown, J. S. L. (2018). Student mental health: some answers and more questions. In Journal of Mental Health (Vol. 27, Issue 3, pp. 193-196). Taylor and Francis Ltd. https://doi.org/10.1080/09638237.2018.1470319

Bruffaerts, R., Mortier, P., Kiekens, G., Auerbach, R. P., Cuijpers, P., Demyttenaere, K., Green, J. G., Nock, M. K., \& Kessler, R. C. (2018). Mental health problems in college freshmen: Prevalence and academic functioning. Journal of Affective Disorders, 225, 97-103. https://doi.org/10.1016/j.jad.2017.07.044

Cova Solar, F., Alvial S, W., Aro D, M., Bonifetti D, A., Hernández M, M., \& Rodríguez C, C. (2007). Problemas de Salud Mental en Estudiantes de la Universidad de Concepción. Terapia Psicológica, 25(2). https://doi.org/10.4067/s0718-48082007000200001

Holm-Hadulla, R. M., \& Koutsoukou-Argyraki, A. (2015). Mental health of students in a globalized world: Prevalence of complaints and disorders, methods and effectivity of counseling, structure of mental health services for students. Mental Health and Prevention, 3(1-2), 1-4. https://doi.org/10.1016/j.mhp.2015.04.003

Idrugo Jave, H. A., \& Sanchez Cabrejos, W. M. (2020). Salud mental en estudiantes de medicina. Investigación En Educación Médica, 33, 107-107. https://doi.org/10.22201/facmed.20075057e.2020.33.19215

Macaskill, A. (2013). The mental health of university students in the United Kingdom. British Journal of Guidance and Counselling, 41(4), 426-441. https://doi.org/10.1080/03069885.2012.743110

Martin, J. M. (2010). Stigma and student mental health in higher education. Higher Education Research and Development, 29(3), 259-274. https://doi.org/10.1080/07294360903470969

Morote-Jayacc, P. V., Sandoval, K. D., Moreno-Molina, M., \& Taype-Rondan, Á. (2020). Mental Health Studies in Medical Students in the Context of COVID-19. In Revista Colombiana de Psiquiatría (Vol. 49, Issue 4, pp. 223-224). Elsevier Doyma. https://doi.org/10.1016/j.rcp.2020.07.005

Okasha, A. (2005). Globalization and mental health: a WPA perspective. World Psychiatry : Official Journal of the World Psychiatric Association (WPA), 4(1), 1-2. https://www.ncbi.nlm.nih.gov/pmc/articles/pmc1414710/

Perales, A., Izaguirre, M., Sánchez, E., Barahona, L., Martina, M., Amemiya, I., Delgado, A., Domínguez, I., Pinto, M., Cuadros, R., \& Padilla, A. (2019). Salud mental en estudiantes de pregrado de la Facultad de Medicina de la Universidad Nacional Mayor de San Marcos. Anales de La Facultad de Medicina, 80(4), 443-450. https://doi.org/10.15381/anales.v80i4.17142 


\section{ESTUDIO DE LA SALUD MENTAL EN ESTUDIANTES UNIVERSITARIOS DE LA RAMA SOCIOSANITARIA}

Rodelo Valle, C., \& Garay Núñez, J. R. (2020). Representaciones sociales de la salud mental y de los trastornos mentales en estudiantes universitarios de enfermería. Dilemas Contemporáneos: Educación, Política y Valores. https://doi.org/10.46377/dilemas.v8i.2483

Valencia Collazos, M. (2007). Trastornos mentales y problemas de salud mental. Día Mundial de la Salud Mental 2007. Salud Mental, 30(2), 75-80. http://www.scielo.org.mx/pdt/sm/v30n2/0185-3325-sm-30-02-75.pdf

Walsemann, K. M., Gee, G. C., \& Gentile, D. (2015). Sick of our loans: Student borrowing and the mental health of young adults in the United States. Social Science and Medicine, 124, 85-93. https://doi.org/10.1016/j.socscimed.2014.11.027

Vélez, L., \& Gutiérrez, J. (2010). Depresión en estudiantes universitarios y su asociación con el estrés académico. CES Medicina, 24. https://revistas.ces.edu.co/index.php/medicina/article/view/1011 